\title{
Topographic Organization of Human Visual Areas in the Absence of Input from Primary Cortex
}

\author{
Heidi A. Baseler, ${ }^{1}$ Antony B. Morland, ${ }^{1,2}$ and Brian A. Wandell ${ }^{1}$ \\ ${ }^{1}$ Psychology Department and Neuroscience Program, Stanford University, Stanford, California 94305, and ${ }^{2 B i o p h y s i c s}$ \\ Section, Physics Department, Imperial College, London SW7 2BZ, United Kingdom
}

\begin{abstract}
Recently, there has been evidence for considerable plasticity in primary sensory areas of adult cortex. In this study, we asked to what extent topographical maps in human extrastriate areas reorganize after damage to a portion of primary visual (striate) cortex, V1. Functional magnetic resonance imaging signals were measured in a subject (G.Y.) with a large calcarine lesion that includes most of primary visual cortex but spares the foveal representation. When foveal stimulation was present, intact cortex in the lesioned occipital lobe exhibited conventional retinotopic organization. Several visual areas could be identified (V1, V2, V3, V3 accessory, and V4 ventral). However, when stimuli were restricted to the blind portion of the visual field,
\end{abstract}

A general view of brain development holds that some aspects of neural development arise without the benefit of experience, whereas others will depend on sensory experience. Within the visual pathways, for example, it has been suggested that the eye-specific domains and perhaps topographic organization within the lateral geniculate nucleus (LGN) and its recipient cortical areas arise early in development without the benefit of visual experience (Shatz, 1996). Hubel and Wiesel (1970) described experience-dependent reorganization of eye-specific domains in LGN and primary visual cortex (V1) during a "critical period" early in development.

Recently, there has been strong evidence for considerable plasticity in primary sensory areas of adult cortex. For example, topographical remapping of sensory space can occur after primary inputs are removed or even in undamaged brains after learning (Buonomano and Merzenich, 1998). Receptive fields in primary visual cortex appear to vary in response to changes in recent stimulus history (Kaas et al., 1990; Heinen and Skavenski, 1991; Gilbert and Wiesel, 1992; Gilbert, 1998).

In this study, we ask to what extent topographical maps in human extrastriate areas reorganize after damage to a portion of primary visual (striate) cortex, V1. Although alternate pathways exist from retina and LGN to extrastriate cortex, their inputs are relatively sparse (Fries, 1981; Yukie and Iwai, 1981; Bullier and Kennedy, 1983; Standage and Benevento, 1983; Kaas and Huerta, 1988). Thus, destruction of V1 eliminates the main source of

\footnotetext{
Received Sept. 4, 1998; revised Dec. 21, 1998; accepted Jan. 8, 1999.

This work was supported by National Institutes of Health National Research Service Award, the Wellcome Trust, and the McKnight Foundation. We thank G. Boynton, J. Demb, and N. Sobel for comments on this manuscript. We thank R. Taylor and T. Aron for developing the gray matter segmentation and rendering software, and S. Chial for her work on cortical unfolding.

Correspondence should be addressed to Heidi Baseler, Department of Psychology, Jordan Hall, Building 420, Stanford University, Stanford, CA 94305.

Copyright (C) 1999 Society for Neuroscience $\quad 0270-6474 / 99 / 192619-09 \$ 05.00 / 0$
}

responses were found primarily in dorsal extrastriate areas. Furthermore, cortex that had formerly shown normal topography now represented only the visual field around the lower vertical meridian. Several possible sources for this reorganized activity are considered, including transcallosal connections, direct subcortical projections to extrastriate cortex, and residual inputs from V1 near the margin of the lesion. A scheme is described to explain how the reorganized signals could occur based on changes in the local neural connections.

Key words: fMRl; retinotopy; visual areas; cortical plasticity; V1; hemianope

visual signals delivered to extrastriate cortical areas and presents an opportunity for considerable reorganization.

We consider three possible consequences of a partial V1 lesion on the responses in extrastriate areas. First, it is possible that extrastriate cortex will be unable to reorganize after the critical period. In this case, large extrastriate regions will remain silent after damage to a portion of V1. Second, it is possible that, despite the removal of the V1 signal, intact regions of extrastriate cortex will retain normal topographic representation based on signals received from alternate subcortical or transcallosal inputs. Third, it is possible that extrastriate cortex has reorganized and can recruit inputs from the spared portion of V1.

We investigated the topographic organization of cortical signals in G.Y., an individual with a large lesion in left anterior calcarine cortex. Based on structural brain images and behavioral testing, the lesion appears to include the peripheral representation of V1, but not the occipital pole, sparing G.Y.'s foveal representation. G.Y. has been studied in a variety of behavioral and neuroimaging experiments, so much is known about his visual abilities (Barbur and Ruddock, 1980; Barbur et al., 1980, 1993; Blythe et al., 1987). A previous study, using positron emission tomography (PET), demonstrated extrastriate activity in G.Y. when moving stimuli were presented to the blind portion of his visual field (Barbur et al., 1993). However, functional area mapping was not done, and the identity of the active regions were estimated using anatomical landmarks.

Using functional magnetic resonance imaging (fMRI), we measured visual responses in G.Y.'s occipital lobes to determine the topography in spared regions of cortex. When visual stimulation included the spared foveal region, the intact portion of G.Y.'s lesioned occipital lobe exhibited conventional topographical organization. Several retinotopically organized visual areas could be identified. However, when stimuli were confined to the blind portion of his visual field, activity was restricted to dorsal extra- 
(A)

Figure 1. Two lesions in G.Y.'s brain. Top images contain a three-dimensional rendering of the cortical surface near the boundary between gray and white matter, obtained from structural MRI data. Bottom images contain single slices from structural data. A, Medial aspect of G.Y.'s left hemisphere. A large lesion is visible near the location ordinarily occupied by calcarine cortex (dashed curve). The sagittal slice below shows the same lesion. $B$, Lateral aspect of G.Y.'s right hemisphere showing the smaller right parietal lesion (dashed curve), with a corresponding coronal image.

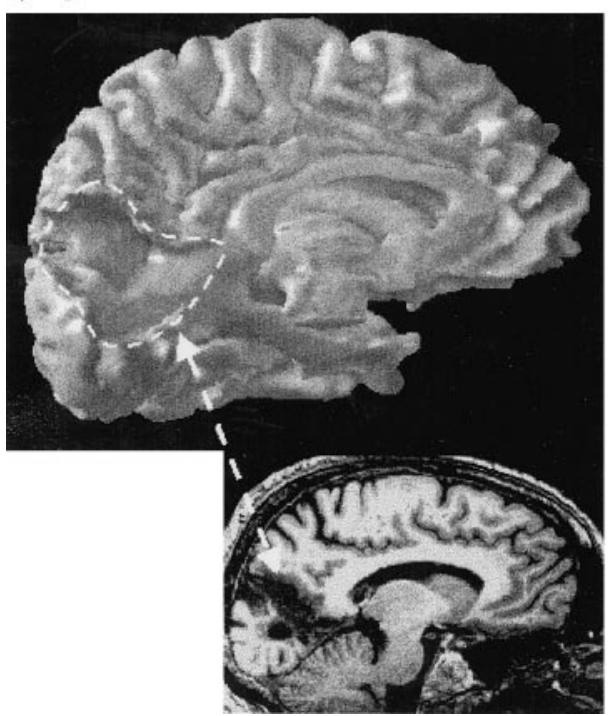

(B)

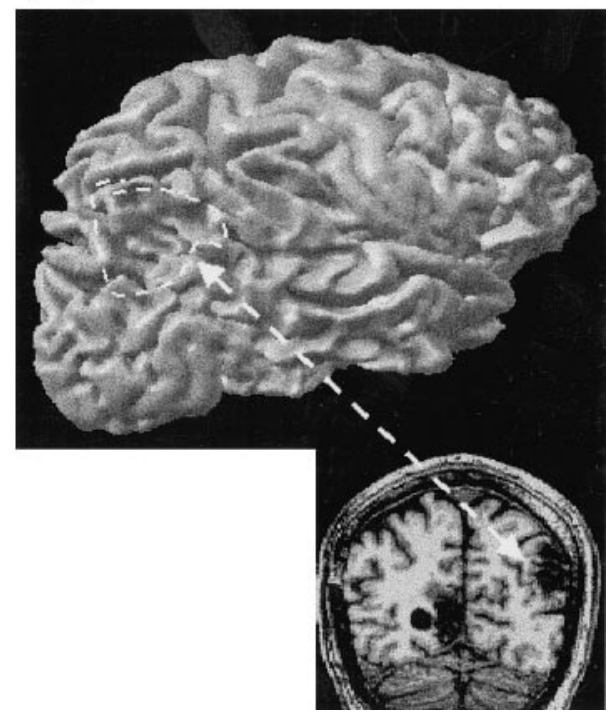

striate areas. Furthermore, these areas exhibited abnormal retinotopic organization. Thus, the same region of cortex appeared to be driven by topographically different inputs, depending on the stimulus. The measurements suggest that human extrastriate cortex maintains some topographical plasticity after cortical lesions.

\section{MATERIALS AND METHODS}

Subjects. Subject G.Y., age 41 years, sustained damage to the posterior part of his brain in a road accident at age 8 years. Structural magnetic resonance images reveal two lesions. One lesion falls on the medial aspect of the left occipital lobe, slightly anterior to the spared occipital pole (Fig. $1 A$ ). The lesion extends dorsally to the cuneus and ventrally to the lingual, but not fusiform, gyrus. The second lesion falls primarily in the inferior parietal lobule and supramarginal gyrus of the right parietal lobe (Fig. 1B). Although the parietal lesion is smaller than the occipital lesion, it was the site of the structural damage caused by the road accident.

Based on tests of visual perimetry, G.Y. appears blind within the right visual field at nearly all eccentricities beyond $2.5^{\circ}$ (homonymous hemianopia with macular sparing) (Barbur and Ruddock, 1980; Barbur et al., 1980, 1993; Blythe et al., 1987; Kentridge et al., 1997). The results of the most recent visual field measurements of G.Y. are shown in Figure 2 (modified from Barbur et al., 1993). Within his blind visual field, however, G.Y. retains a range of residual visual capacities, although all aspects of his vision are significantly impoverished compared with normals. His residual vision includes some ability to discriminate color (Brent et al., 1994) and orientation (Morland et al., 1996). G.Y. is particularly sensitive to visual motion and transient stimuli (Barbur and Ruddock, 1980; Barbur et al., 1980, 1993). Residual vision to motion has been reported in other cases of cortical blindness (Blythe et al., 1987; Celesia et al., 1991; Perenin, 1991).

Visual behavioral responses to stimuli in G.Y.'s left hemifield are normal, despite the right parietal lesion. His right occipital lobe appears to have suffered no damage in structural MRI records. Therefore, we used responses measured in his right occipital lobe as a control to compare with those measured in his lesioned left occipital lobe. Subject A.P., who has no known lesions, also served as a control.

Both subjects were highly experienced at tasks requiring good visual fixation. Previous studies have shown that G.Y. can fixate reliably, at least for short periods: within 20-40 min of arc over several seconds (Barbur et al., 1993; Weiskrantz et al., 1995; Kentridge et al., 1997). A recent study confirmed that experienced observers can also fixate quite accurately over extended periods, such as those used in neuroimaging experiments (Lucas et al., 1996).

Data acquisition. Magnetic resonance images were acquired using a 1.5 T GE Signa scanner and special purpose head coil or a surface coil placed at the back of the head. Images were collected in eight planes oriented

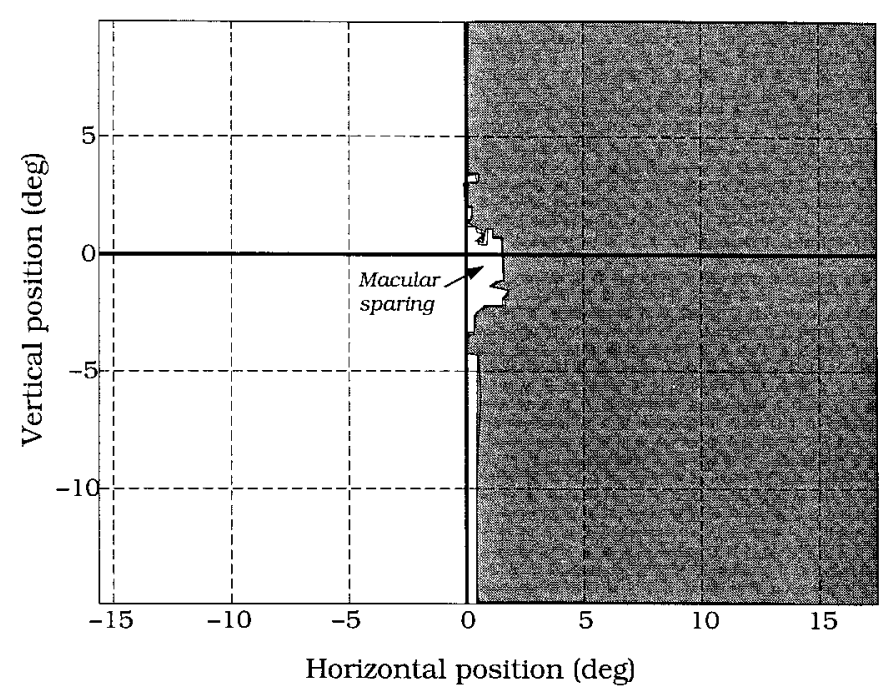

Figure 2. Measurement of G.Y.'s visual field sensitivity, using specialized visual perimetry. White area indicates normal sensitivity; gray area indicates region of visual field loss. (Modified from Barbur et al., 1993.)

either perpendicular or parallel to the calcarine sulcus. Functional images, measuring a blood oxygenation level-dependent signal (Ogawa et al., 1990), were acquired every $3 \mathrm{sec}$ using a two-shot, two-dimensional spiral gradient-recalled echo sequence (echo time/repetition time/flip angle, $40 \mathrm{msec} / 1500 \mathrm{msec} / 90^{\circ}$; voxel size, $1 \times 1 \times 4 \mathrm{~mm}$; effective spatial resolution, $1.5 \times 1.5 \times 4 \mathrm{~mm}$ ) (Meyer et al., 1992; Glover and Lai, 1998). Structural (T1-weighted) images were acquired in the same planes and resolution as the functional images to coregister the functional and anatomical data.

Retinotopic mapping. Human primary visual cortex and several other nearby visual areas are retinotopically organized. To locate and identify early visual areas in occipital cortex, we used a mapping procedure that takes advantage of the retinotopic organization of these areas (Engel et al., 1994, 1997; Sereno et al., 1995; DeYoe et al., 1996).

A slowly expanding annulus was used to map the eccentric representation. The annulus comprised a black and white checkerboard contrast pattern presented on a gray background (Fig. 3B, inset). The checkerboard contrast pattern flickered at $7.4 \mathrm{~Hz}$. Subjects fixated while the annulus expanded from the central fixation point to either 8 or $13^{\circ}$ of eccentricity once every $36 \mathrm{sec}$. Check size varied slightly with eccentric- 
ity; checks were about $1^{\circ}$ of visual angle in the eccentric dimension and ranged from 0.3 to $2.1^{\circ}$ of visual angle ( $15^{\circ}$ of polar angle) in the angular dimension. The annulus traveled from fixation to periphery seven times during each scan (total time, $7 \times 36=252 \mathrm{sec}$ ). Data from the first cycle were discarded to avoid early transients in the fMRI signal. Brain regions processing a particular position in the visual field were activated when the reversing check pattern passed through that position.

A rotating wedge (Fig. $3 A$, inset) was used to map angular position in the visual field. The checkerboard pattern inside the $90^{\circ}$ wedge reversed at $6.66 \mathrm{~Hz}$. Other temporal parameters (rotation rate, number of cycles, etc.) were similar to those used to map eccentricity. Several retinotopically organized visual areas [V1, V2, V3, V3 anterior (V3a), and V4 ventral $(\mathrm{V} 4 \mathrm{v})]$ in the normal human brain abut one another at the vertical and horizontal meridian representations. Boundaries between these areas can be identified from the positions of the horizontal and vertical meridians measured using the rotating wedge stimulus.

In both G.Y. and a normal control, measurements were made using two different rotating wedge patterns. In the "full wedge" condition, the stimulus spanned a radius of $8^{\circ}$ of visual angle. In the "annular wedge" condition, the wedge was confined to the blind region of his right visual field, spanning only the eccentricities between 4 and $8^{\circ}$ from the fovea (Fig. 3C, inset). Our strategy was to identify visual area boundaries using the full wedge stimulus and then compare the activity caused by stimulation within the blind visual field. In this way, we could compare the activity driven primarily by the LGN-to-V1 pathway (full wedge) with activity driven by the secondary spared visual pathways (annular wedge).

Data analysis. The correlation and phase of the fMRI signal were measured using the methods described by Engel et al. (1997). Briefly, the Fourier transform of the fMRI signal time series was calculated for each voxel of the functional images. The amplitude and phase of the time series harmonic at the fundamental stimulus frequency were measured. The correlation was calculated as the square root of the squared amplitude of the response at the stimulus frequency divided by the sum of the squared amplitudes at all frequencies.

The phase of the fMRI signal can be used to identify the angular (or eccentric) position of the stimulus causing the response. To relate the response phase of the fMRI signal to the stimulus position, it is necessary to account for the response delay inherent in the hemodynamics, a delay on the order of 4-6 sec (Boynton et al., 1996). The stimulus alternation at a given point in the visual field is a temporal square wave with a $25 \%$ duty cycle. The phase of the square wave fundamental is advanced approximately $\pi / 4$ radians, corresponding to $\sim 4.5 \mathrm{sec}$ for a period of 36 sec. Because the phase advance of the stimulus fundamental compensates for hemodynamic delay, the phase of the fMRI signal can be used without modification to estimate the stimulus position.

Cortical unfolding. We computationally unfolded the occipital cortex of each observer to visualize functional activity across the cortical sheet and to identify the retinotopic visual areas (Engel et al., 1997). Flattening was performed based on high-resolution structural three-dimensional volumes measured in each subject's brain (voxel size, $0.9 \times 0.9 \times 1.2$ $\mathrm{mm}$ ). Gray matter was segmented (Teo et al., 1997) within the images and then unfolded into a two-dimensional representation of the cortical surface using publicly available software (http://white.stanford.edu). Functional data were aligned to the high-resolution volumes and projected onto the flattened gray matter, creating two-dimensional phase and correlation maps.

The data shown in all figures were spatially blurred by convolution with a Gaussian kernel to improve the signal-to-noise ratio of the measurements. The Gaussian kernel had an SD of $1.4 \mathrm{~mm}$ (support of $10 \times 10 \mathrm{~mm}$ ). The spatial averaging takes place along the cortical surface and thus only includes measurements that arise within the cerebral cortex. By averaging within the flattened representation, signals outside the gray matter are suppressed.

\section{RESULTS}

\section{Retinotopic organization within occipital cortex}

The retinotopic organization and arrangement of several visual areas in the left occipital lobe of a normal control are shown in Figure 3. Each panel represents the data acquired from eight parallel planes in the left occipital lobe. The data from the separate planes were integrated into a single image by computationally unfolding the gray matter and superimposing the measured fMRI signal onto the flattened representation of cortex.
The colors in Figure 3 denote the location within the visual field of the stimulus that caused the activity. The association between the representation of the visual field and color are shown by the colored legends near each panel; the gray icons indicate the stimulus type. The colored regions show activity levels in which the correlation between the response and stimulus fundamental frequency exceeded 0.20 . For all thresholds above this level, the images are consistent with the one shown. Below this correlation level, the images appear noisy, and the spatial pattern of angular positions takes on a patchy, random appearance.

The patchiness near the foveal representation in the eccentricity map in Figure 3 may have been a result of the reduced resolution parallel to the calcarine. In the control subject, slices were oriented perpendicular to the calcarine sulcus to optimize resolution for mapping polar angle and visual area identification. In G.Y., however, slices were oriented parallel to the calcarine when mapping eccentricity and perpendicular to the calcarine when mapping polar angle.

The retinotopic organization of G.Y.'s intact right occipital lobe (data not shown) was similar to that of the normal subject, A.P., and to other measurements in our lab and by others using similar methods (Engel et al., 1997; Smith, et al., 1998).

Figure 4 shows the retinotopic mapping in the unfolded, left lesioned occipital lobe in G.Y. Figure $4 A$ shows responses to the full wedge. There is a great deal of spatially organized activity, with the upper quarter field represented in the ventral aspects and the lower quarter field represented in the dorsal aspects. Boundaries between several areas are plainly visible where the angular representation reverses direction. This stimulus reveals that the residual cortex in the lesioned occipital lobe has a conventional retinotopic organization and includes dorsal and ventral portions of V2 and V3. Portions of V3a and V4v can also be seen. Activity in the spared portion of $\mathrm{V} 1$ is less extensive and somewhat more disorganized than in the normal (Fig. $3 A$ ). However, it is not certain whether this difference is significant or whether it is attributable to partial volume effects by the relatively large functional voxels spanning the narrow calcarine sulcus.

Figure $4 B$ shows responses to the expanding ring stimulus. The activity is consistent with the macular sparing evident in G.Y.'s visual perimetry maps; most of the activity represents eccentricities $<2.5^{\circ}$. Beyond this eccentricity, activity is patchy or absent.

Figure $4 C$ shows responses to the annular wedge stimulus. The annular wedge fell in the periphery, beyond the macular sparing in the right hemifield. The responses are more fragmented than they were to the full wedge, and the reduction is particularly striking in area V1. The upper visual field representations in V2v, $\mathrm{V} 3 \mathrm{v}$, and $\mathrm{V} 4 \mathrm{v}$ show relatively little activity. However, there is significant activity, particularly in the lower visual field representations of dorsal V2 (V2d), dorsal V3 (V3d), and V3a, in the region beyond the $2.5^{\circ}$ isoeccentricity contour marking G.Y.'s macular sparing. The flat map of G.Y.'s lesioned occipital lobe reveals that the lesion has removed more of the peripheral representation in ventral than in dorsal visual areas.

In the annular wedge condition, nearly all of the activity falls within a narrow range of phase corresponding to angular positions around the lower vertical meridian. Even portions of the brain that showed ordinary responses when presented with the full wedge responded at this abnormal phase in the annular wedge condition.

In addition, activation in response to the annular wedge can be observed just below the ventral boundary of V4v (Fig. 4C). We have observed a representation of the lower visual quadrant in 
(A)

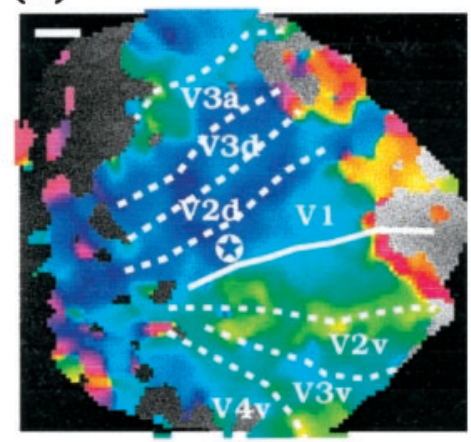

(B)
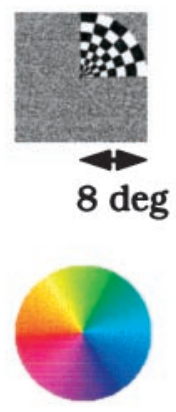
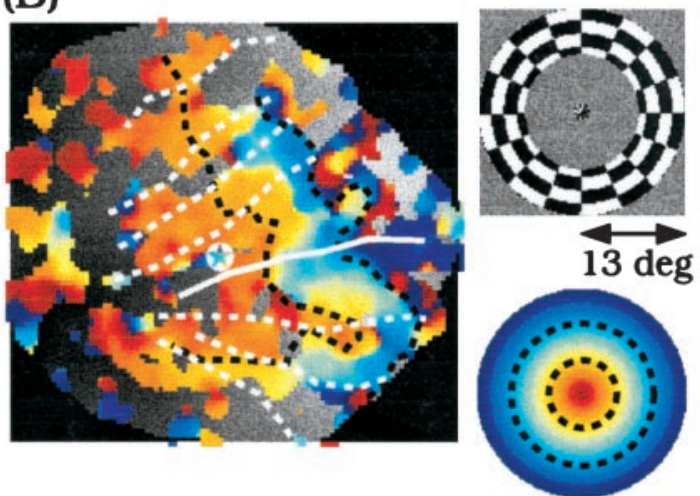

(C)
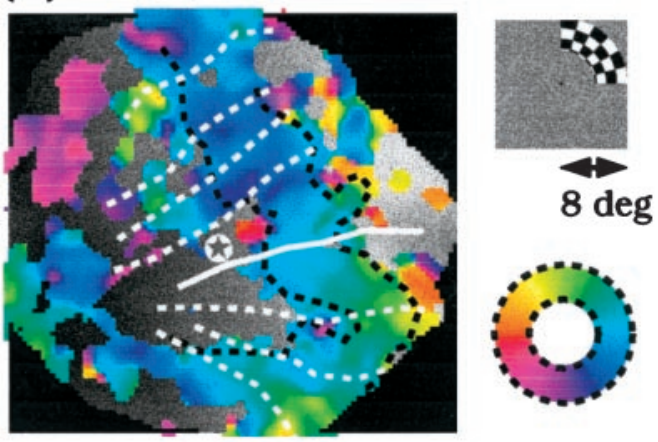

(A)

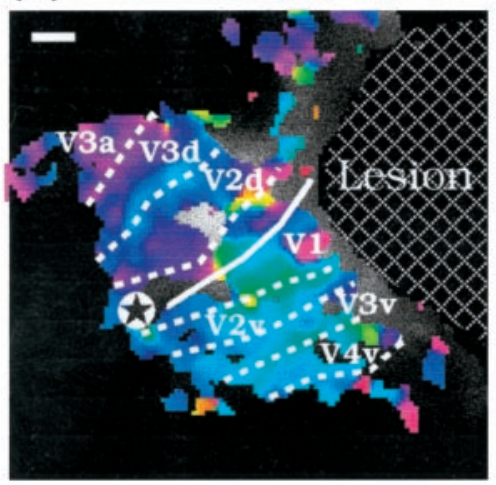

(B)

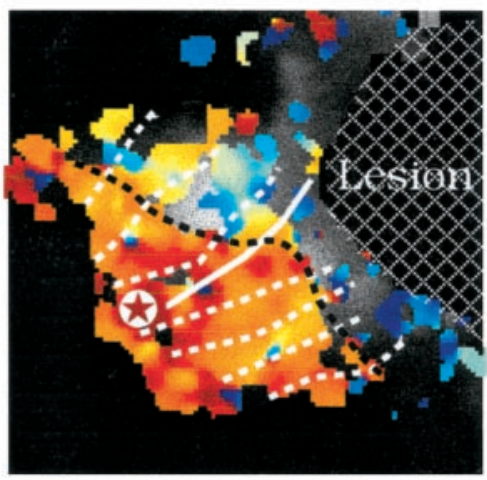

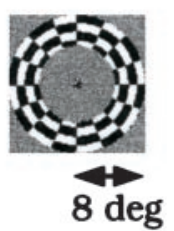

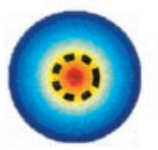

(C)
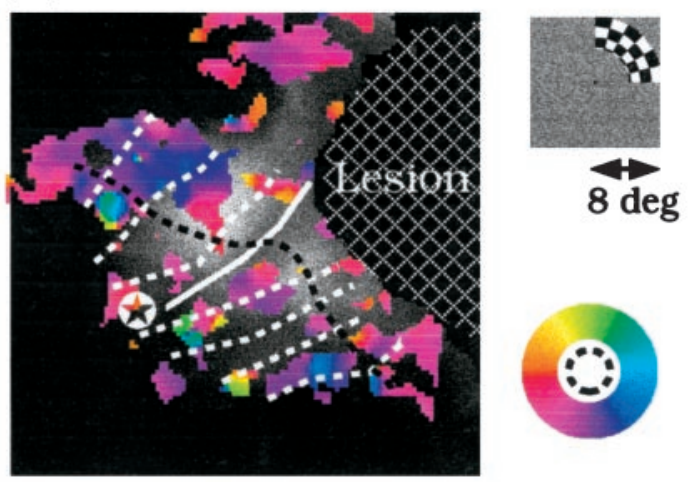

Figure 3. Top. Representations of a control subject's (A.P.) unfolded left occipital cortex. The underlying gray scale image, which is mainly obscured by the overlaid activity, represents the unfolded cortex. In all images in this and Figure 4, dorsal is up, ventral is down, posterior is left, and anterior is right. The gray scale represents the lateral-medial dimension; dark pixels originate from lateral parts in the three-dimensional folded cortex, and light pixels originate from medial parts. Scale bar (in A), $1 \mathrm{~cm}$. Anatomical landmarks are indicated by the star (occipital pole) and the solid white line (fundus of the calcarine sulcus). The color overlay in each image represents the stimulus position that caused activity at each point in (Figure legend continues) 
this region in the retinotopic flat maps of many of our normal subjects. Other fMRI studies have shown a representation of the lower visual quadrant in ventral cortex, claiming it as a part of V4 (McKeefry and Zeki, 1997) or part of an entirely new area, V8 (Hadjikhani et al., 1998). Again, these signals are consistent with the preservation of the lower vertical meridian representation in G.Y.

Responses in G.Y.'s intact occipital lobe were consistent with retinotopic measurements in the right occipital lobe of the normal observer (data not shown). Signals in V1 were strongly driven by stimulus phases spanning $180^{\circ}$, representing the contralateral hemifield. Signals in dorsal and ventral extrastriate visual areas V2 and V3 were highly correlated with stimuli spanning $90^{\circ}$ phase, corresponding to lower and upper quadrants of the visual field, respectively.

\section{Phase Analyses}

In this section, we provide a quantitative analysis of the data to determine more precisely which portions of the visual field are represented by the abnormal signals. Figures 5-8 show voxel frequency histograms as a function of angular position for different visual areas and for the two different rotating wedge conditions. Each histogram bar shows the number of voxels that exceeded the estimated noise level, a stimulus correlation of 0.2. Correlations of all the voxels in each angular position bin (including those $<0.2$ ) were also averaged. The mean correlation was then calculated across all bins containing at least 20 voxels exceeding the 0.2 noise threshold.

The distribution of angular positions represented by voxels in each visual area in G.Y.'s left lesioned occipital lobe is shown in Figure 5. Visual areas were identified using the response pattern to the full wedge stimulus (Fig. 4A). In Figure 5, the top row represents responses in the full wedge condition, and the bottom row represents responses to the annular wedge.

In the lesioned occipital lobe, response phases were generally consistent with retinotopic representations of the visual field when G.Y. viewed the full wedge, although there were relatively few voxels responding to phases driven by visual field positions near the upper vertical meridian (Fig. 5A).

The responses to the annular wedge, however, were quite different. Visual areas V1 and ventral regions of V2 and V3 displayed essentially no organized activity. Large signals were observed, however, in dorsal extrastriate visual areas V2 and V3 (also in V3a; data not shown). Hence, the clusters of responses around the lower vertical meridian in the pooled data (Fig. 5B) are derived from dorsal and not ventral V2 and V3. The responses differ from normal controls in that (1) there is a reduced response to the horizontal meridian, and (2) the responses represent po-

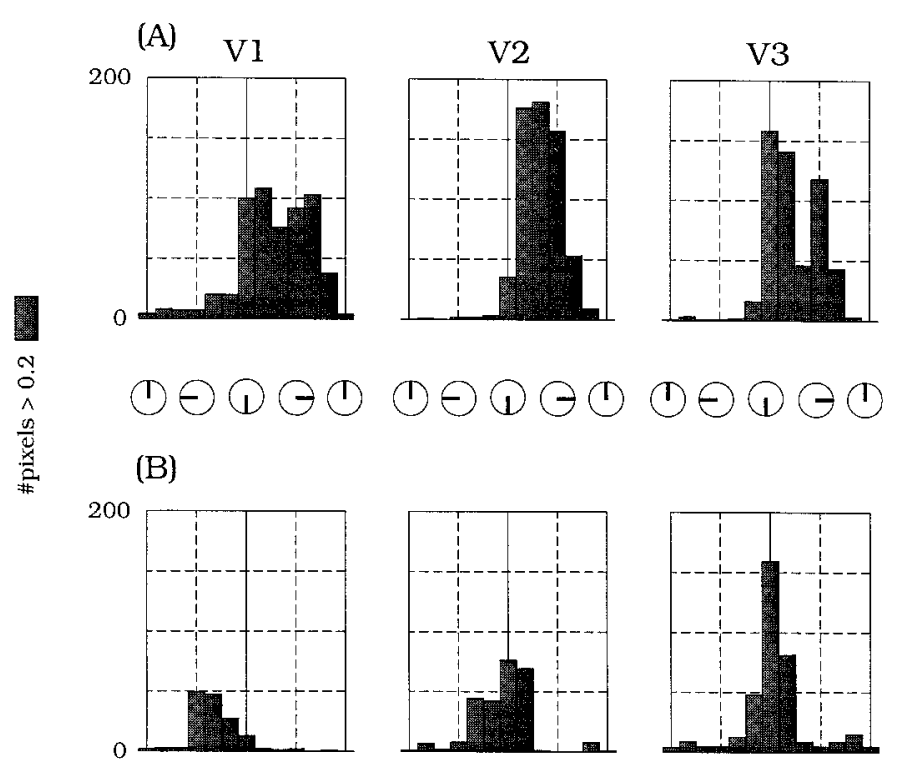

Figure 5. Summary of the fMRI responses within individual visual areas to the two types of rotating wedge stimulus in G.Y.'s left (damaged) occipital lobe. Each column summarizes responses from a different visual area: V1, V2, or V3. Dorsal and ventral portions of V2 and V3 are combined here. The horizontal axis represents angular position of the visual field representation as inferred from the phase of the fMRI signal. The vertical axis indicates the number of voxels exceeding the 0.2 stimulus correlation level for each histogram bin. $A$, Measurements using the full wedge stimulus. Mean correlations were as follows: V1, 0.33; V2, 0.33; V3, 0.33. (See Results, Phase analyses, for explanation of mean correlation calculation.) $B$, Measurements using the annular wedge stimulus. Mean correlations were as follows: V1, 0.16; V2, 0.22; V3, 0.26.

sitions that extend into the ipsilateral hemifield across the lower vertical meridian.

Response positions represented in the visual areas in the left occipital lobe of the control subject, A.P., are shown for comparison in Figure 6. Note the similarity in the distributions between the two stimulus conditions (Fig. 6A,B) in the normal subject.

Response positions represented in the visual areas of G.Y.'s right, nonlesioned occipital lobe are shown in Figure 7. Although fewer voxels respond to the annular $(B)$ than to the full wedge stimulus $(A)$, the distribution of angular positions represented is similar in the two conditions. In both conditions, responses in the right, intact occipital lobe represent the contralateral left hemifield.

Figure 8 shows histograms comparing activity in the full and annular wedge conditions in dorsal V2 and V3 with ventral V2 and V3. Both dorsal and ventral cortex respond to the appropriate

\section{$\leftarrow$}

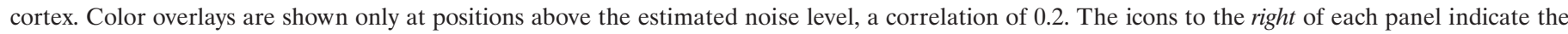

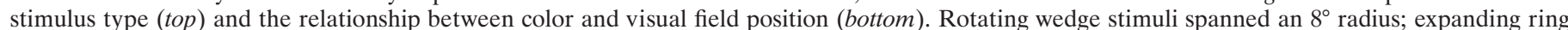

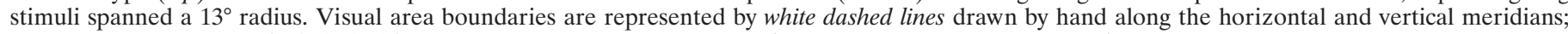

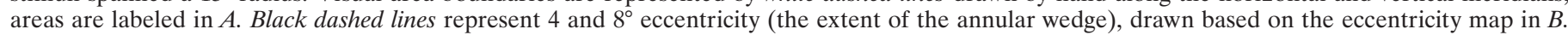

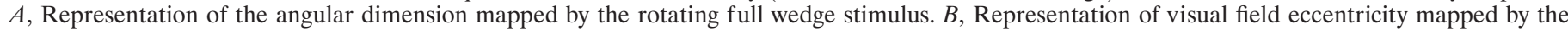
expanding ring stimulus. $C$, Representation of the angular dimension mapped by the rotating annular wedge stimulus.

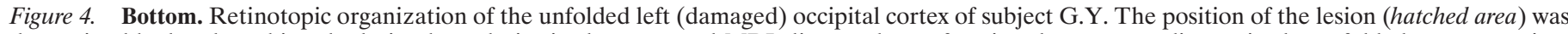

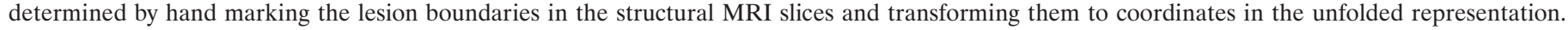

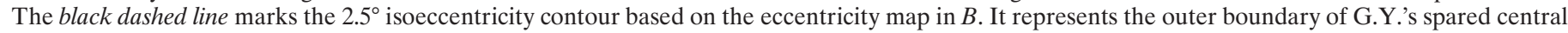

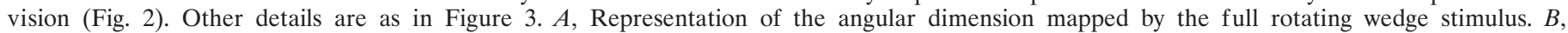

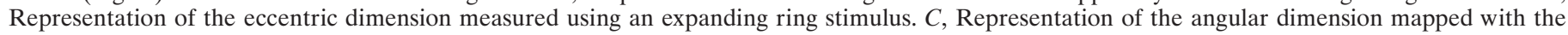
annular rotating wedge stimulus. 


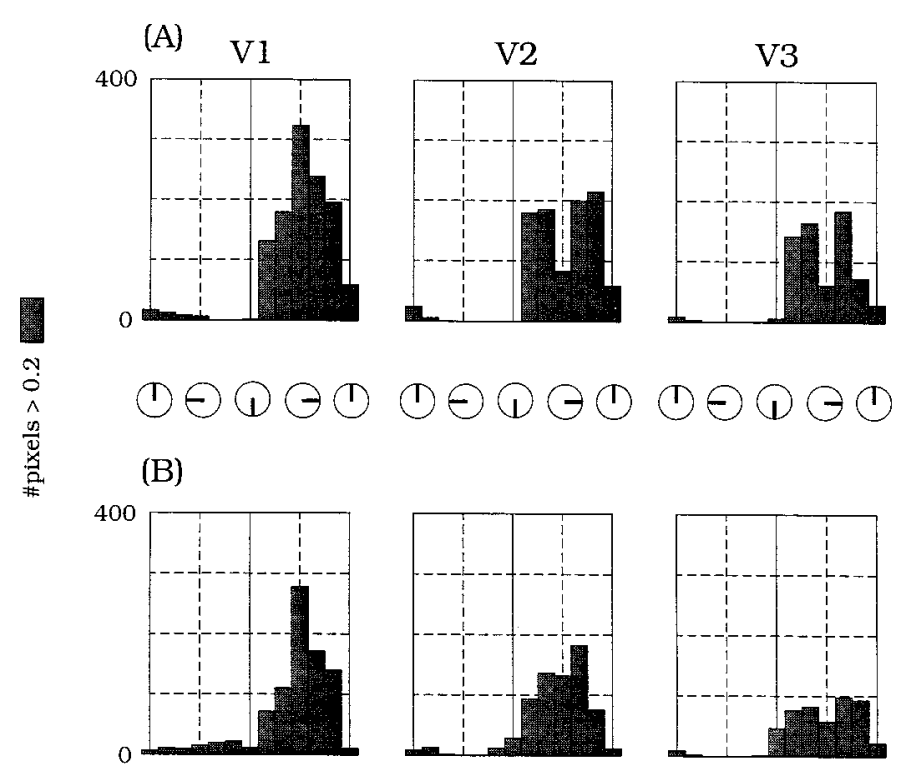

Figure 6. Summary of the fMRI responses within individual visual areas to the two types of rotating wedge stimulus in the left occipital lobe of the control subject A.P. Details are as in Figure 5. A, Measurements using the full wedge stimulus. Mean correlations were as follows: V1, 0.52; V2, 0.46; $\mathrm{V} 3,0.45$. $B$, Measurements using the annular wedge stimulus. Mean correlations were as follows: V1, 0.30; V2, 0.38; V3, 0.31.

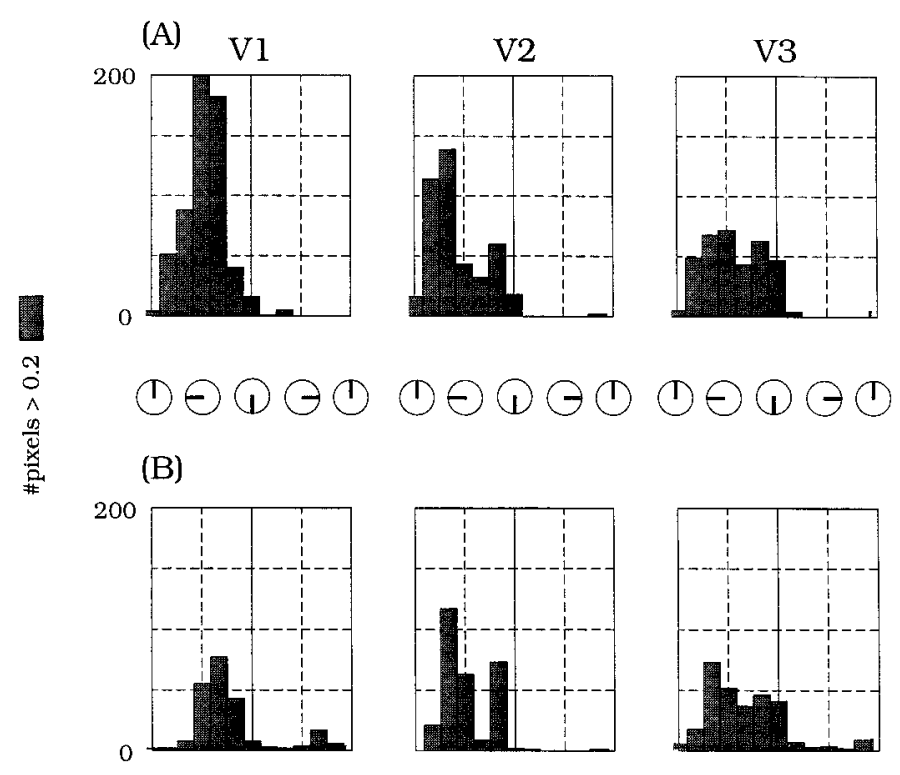

Figure 7. Summary of the fMRI responses within individual visual areas to the two types of rotating wedge stimulus in the nonlesioned right occipital lobe of G.Y. Details are as in Figure 5. $A$, Measurements using the full wedge stimulus. Mean correlations were as follows: V1, 0.35; V2, $0.38 ; \mathrm{V} 3,0.36$. $B$, Measurements using the annular wedge stimulus. Mean correlations were as follows: V1, 0.22; V2, 0.29; V3, 0.29 .

visual field quadrants in the full wedge condition (Fig. 8A,C). However, only dorsal V2 and V3 respond to the annular wedge (Fig. $8 B$ ). Responses to the annular wedge were again confined to the vertical meridian representation (Fig. $8 B$ ). Dorsal and ventral areas in G.Y.'s intact right occipital lobes and in both occipital lobes of the control subject, A.P., responded to the appropriate contralateral quadrants for both wedge stimuli (data not shown).

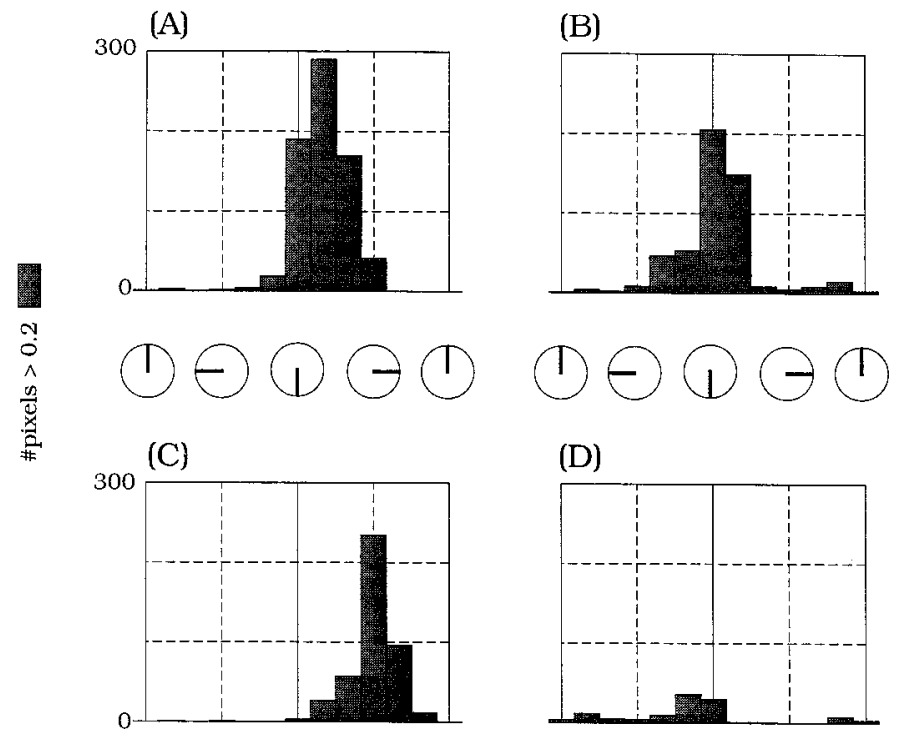

Figure 8. Comparison of the fMRI responses within dorsal and ventral areas to the two types of rotating wedge stimulus in G.Y.'s left (lesioned) occipital lobe. Responses from areas V2 and V3 are combined in each histogram. $A$, Dorsal responses, full wedge stimulus. $B$, Dorsal responses, annular wedge stimulus. $C$, Ventral responses, full wedge stimulus. $D$, Ventral responses, annular wedge stimulus. Other features are as in Figure 5.

\section{Comparison of annular and full wedge responses}

The extrastriate activity caused by the annular wedge does not conform to conventional retinotopic positions (Fig. $4 C$ ). Figure 9 shows the nature of the abnormal signals by making a direct comparison between the full and annular wedge responses. Each point represents the angular visual field representation of a single voxel that was active in both the full wedge and annular wedge experiments.

In the control subject, the angular representation is the same in the two stimulus conditions (Fig. $9 A, B$ ). The points fall close to a line with unit slope. Moreover, as expected, voxels in each occipital lobe represent an entire contralateral hemifield. In G.Y.'s intact occipital lobe, the data are similar to the normal control (Fig. 9C).

In G.Y.'s lesioned occipital lobe (Fig. 9D), the angular representations in the two stimulus conditions differ. The collection of points falls significantly below the line of unit slope. The voxels active in both conditions are restricted to positions in the lower right quadrant of the visual field, normally represented in the dorsal visual areas. The data show that the same region of cortex in G.Y.'s lesioned occipital lobe responds differently depending on the stimulus configuration. Hence, the annular wedge stimulus causes activity at cortical locations that are inconsistent with normal retinotopic organization.

\section{Summary}

We can summarize our measurements of the normal control, G.Y.'s intact occipital lobe, and G.Y.'s lesioned occipital lobe with the following points. (1) Stimulation within spared regions of the visual field produced normal retinotopic maps in G.Y.'s intact right occipital lobe and in the intact foveal representation of the lesioned left occipital lobe. Several visual areas could be identified in both lobes, including V1, V2, V3, V3a, and V4v. (2) The lesion in G.Y.'s left occipital lobe extends not only into the peripheral representation of $\mathrm{V} 1$ but also into the peripheral 
(A)

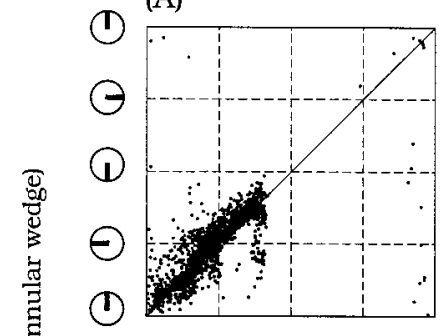

(C)

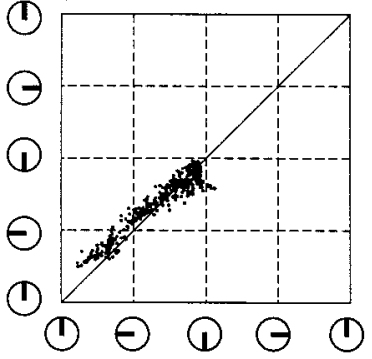

(B)

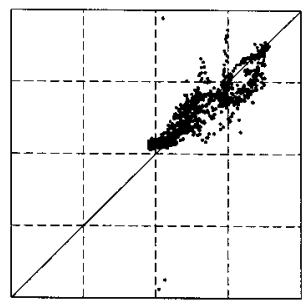

(D)

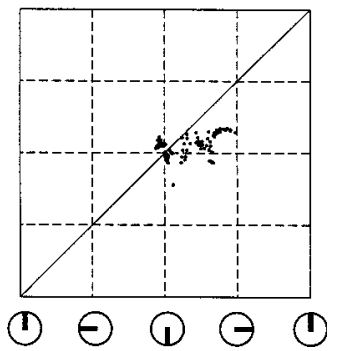

Angular position (full wedge)

Figure 9. Comparison of angular visual field representation measured in the full (horizontal axis) and annular (vertical axis) wedge conditions. Voxels exceeding a correlation threshold of 0.33 in both stimulus conditions are shown. Responses from V1, V2, and V3, dorsal and ventral, and $\mathrm{V} 3 \mathrm{a}$ and $\mathrm{V} 4 \mathrm{v}$ are pooled in each graph. The identity line is plotted for comparison. Because of the cyclic nature of the angular dimension, points falling at the far right (top) margin of the graph also could be plotted at the far left (bottom) margin. A, Control subject A.P., right hemisphere; $B$, control subject A.P., left hemisphere; $C$, G.Y., right hemisphere; $D$, G.Y., left hemisphere.

representations of ventral extrastriate areas $\mathrm{V} 2 \mathrm{v}, \mathrm{V} 3 \mathrm{v}$, and $\mathrm{V} 4 \mathrm{v}$. Hence, stimulation restricted to the blind portion of the visual field generated activity within the lesioned occipital lobe primarily in dorsal extrastriate areas V2d, V3d, and V3a. (3) Stimuli restricted to the blind portion of the visual field in G.Y. generated response patterns that were inconsistent with normal retinotopic organization. Extrastriate areas that had shown normal organization when the fovea was also stimulated now responded primarily to positions near the lower vertical meridian.

\section{DISCUSSION}

\section{Related results}

A previous neuroimaging (PET) study using moving stimuli in G.Y.'s blind field has demonstrated significant activation at several cortical positions (Barbur et al., 1993). Activity was reported at the lateral junction of the occipital and parietal lobes (human $\mathrm{MT}+/ \mathrm{V} 5$, a motion-responsive region analogous to macaque area $\mathrm{MT} / \mathrm{V5}$ ) and also in area 7. In addition, an active location in the occipital lobe, thought to be V3, was observed. It has been suggested that the activity in MT + may be caused by a direct projection from the dorsal LGN to $\mathrm{MT}+$, although there is some disagreement on this point (Rodman et al., 1989; Hotson et al., 1994; ffytche et al., 1996).

Human area MT+ is not retinotopically organized at the fMRI signal resolution. Consequently, the stimuli used in the present experiments did not produce significant activity in MT+. In separate experiments, however, we have confirmed the presence of significant activity in MT+ when G.Y. observed moving targets. Our acquisition planes did not extend to area 7.

The PET activations found by Barbur et al. (1993) were ob- tained at a relatively coarse spatial resolution, but the reported activation of V3 in the superior occipital lobe was qualitatively in the position of dorsal V3 measured here. The region activated in the PET study extends to a position that is anterior to our measurement of dorsal V3, but this can be explained in part by the fact that Barbur et al. used more eccentric stimuli $\left(11^{\circ}\right)$ than those used in this study. Contrary to our results, however, Barbur et al. (1993) generated activity in putative dorsal V3 with a stimulus that was restricted to the upper visual field. V3d in either hemisphere normally represents the lower visual quadrants. However, adjacent area V3a has been shown to contain both the upper and lower visual quadrants (Tootell et al., 1997).

Our measurements extend those of Barbur et al. (1993) in several ways. First, we have measured the location of several retinotopically organized visual areas. This has enabled us to unambiguously identify both the position of the lesion and residual neural responses with respect to functionally defined areas. Second, we have shown that residual activity is present in V2d, $\mathrm{V} 3 \mathrm{a}$, and ventral to $\mathrm{V} 4 \mathrm{v}$, in addition to dorsal V3. Third, we have provided more precise information about the representations of the visual field in the lesioned occipital lobe. Specifically, we have shown that the topography of extrastriate areas in G.Y. depends on the stimulus configuration.

\section{Source of the blind hemifield signals}

Next, we consider three possible sources of the signals we have measured in G.Y.'s lesioned occipital lobe.

First, two subcortical pathways project directly to extrastriate visual areas in monkey. One pathway is from the pulvinar (via the superior colliculus) and a second is from the LGN. Anatomical studies in primate have shown that both the LGN (Fries, 1981; Yuki and Iwai, 1981; Bullier and Kennedy, 1983) and pulvinar projections (Kaas and Huerta, 1988) are sent to many areas of extrastriate cortex. After deactivation of V1 by cooling, singleunit measurements failed to find activity in V2, V3, and V4 but did find activity in area V3a (Schiller and Malpeli, 1977; Girard and Bullier, 1989; Girard et al., 1991a,b). Because the direct projections from subcortical structures to extrastriate cortex are sparse, extrastriate activity may be difficult to detect during single-unit electrode recordings.

Direct subcortical projections have been proposed as the source of signals in residual vision (Weiskrantz et al., 1974; Barbur et al., 1980, 1993; Blythe et al., 1987). However, because these subcortical structures are organized retinotopically and represent the entire visual field, it seems unlikely that they would generate activity in G.Y. only near the lower vertical meridian (Berson and Stein, 1995).

A spared strip of dorsal V1 beyond the macular representation is a second possible source for the signals in the lesioned occipital lobe. Barbur et al. (1993) also made a detailed perimetric map of G.Y.'s visual field (Fig. 2). In addition to the macular sparing, their map reveals a thin strip of spared vision in the right visual field along the lower vertical meridian. We were unaware of this observation until after our fMRI measurements were made, because it was not noted in the text or in other behavioral studies in G.Y. Furthermore, an earlier visual field map measured using static light stimuli failed to show a spared strip along the lower vertical meridian (Barbur et al., 1980). Note that the spared strip corresponds with the representation of residual activity in left extrastriate cortex found in our data.

Our measurements do not support a residual V1 source for two reasons. First, there is only a fragmented and inconsistent re- 
sponse beyond the macular representation in the flattened representation (Fig. 4). Second, the quantitative analyses of V1 using histograms show little V1 activity corresponding to the lower vertical meridian (Fig. $5 B$ ). Still, this region of V1 may contain a weak and spatially disorganized representation of the normal LGN signals that are difficult to detect using the phase-encoding methods.

Transcallosal fibers are a third possible source of the signals in the lesioned occipital lobe. In both monkey and human, the callosal fibers connecting early retinotopically organized visual areas represent the vertical meridian (Zeki, 1970; Bunt and Minckler, 1977; Bunt et al., 1977; Van Essen et al., 1982; Clarke and Miklossy, 1990). Tootell et al. (1998) have confirmed the presence of ipsilateral activity, presumably mediated by callosal connections, in several retinotopically organized human visual areas. The activity in the lesioned occipital lobe, which is restricted to the lower vertical meridian, is consistent with a callosal source. The difference between the ventral and dorsal activity levels may be explained by the fact that the lesion lies somewhat ventral; dorsal fibers may be relatively spared. A psychophysical study in G.Y. has reported that he senses motion toward the vertical meridian in his blind visual field when moving stimuli are presented to his normal visual field (Finlay et al., 1997).

\section{Cortical reorganization}

Several recent reports have suggested a large range of plastic behavior within the human brain (Gilbert, 1996, 1998; Karni and Bertini, 1997; Buonomano and Merzenich, 1998). The highly detailed measurements we have obtained from G.Y.'s brain support the presence of plasticity in intact portions of the lesioned occipital lobe. Figure $4 A$ shows that when foveal representations are activated, nearby peripheral representations in V2d and V3d exhibit normal angular topography. Figure $4 C$ shows that when foveal stimulation is absent, the same regions in $\mathrm{V} 2 \mathrm{~d}$ and $\mathrm{V} 3 \mathrm{~d}$ now represent positions near the lower vertical meridian. These regions, which have been severed from their usual direct input, appear to be colonized by neighboring cortex that continues to receive input.

Figure 10 provides a framework for understanding how the same portion of cortex can respond differently in the full and annular wedge conditions. The data are explained assuming only changes in the relative significance of local connections. For simplicity, the figure shows only the signals in V1 and dorsal V2, although the same principle also applies to signals in dorsal V3.

In intact cortex, dorsal V2 neurons receives its primary input from corresponding retinotopic representations in V1 (Fig. 10A). In G.Y.'s lesioned occipital lobe, however, these inputs are absent. We assume that when V2 neurons are deprived of their normal V1 input, they are colonized by other neurons in neighboring cortex (Fig. 10B). The colonization may be mediated by strengthening or disinhibition of long-range connections or by the creation of new connections (Das and Gilbert, 1995). In Figure 10, these new connections are indicated by bold arrows.

The development of these new connections explains G.Y.'s measurements as follows. In the full wedge condition (Fig. 4A), neurons representing the fovea drive the nearby peripheral representations and generate a normal representation of angular visual field position. In the annular wedge condition, neurons representing the fovea are not stimulated. Instead, nearby neurons that represent the peripheral portion of the lower vertical meridian generate an abnormal representation of the visual field position (Fig. 4C). Finally, when measured using an expanding
(A)
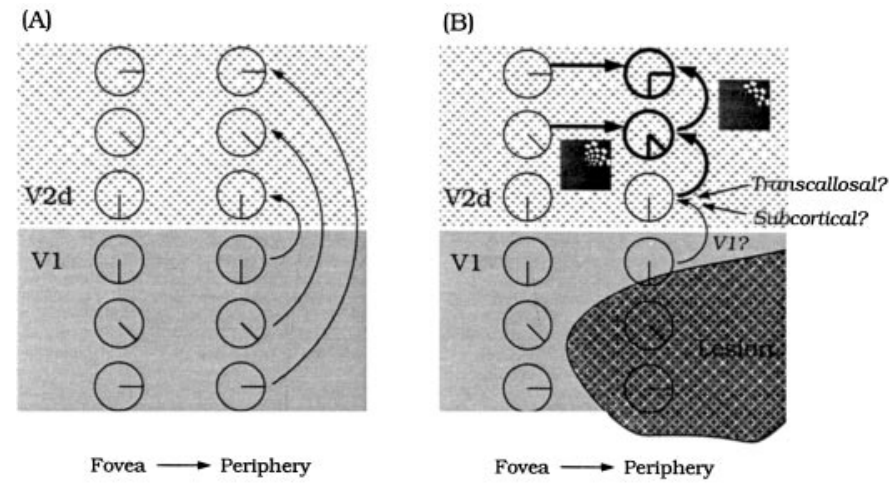

Figure 10. A framework for explaining G.Y.'s cortical responses. For simplicity, only the connectivity between V1 and V2d is illustrated. In both panels, the shaded gray region represents V1, and the lightly hatched area represents V2d. Circles with lines describe the angular representation at different cortical locations. The fovea is represented on the left and periphery on the right in each panel. A, Intact occipital lobe. Primary, feed-forward inputs to V2d are shown as thin arrows. $B$, Lesioned occipital lobe in G.Y. The lesion is indicated by the dark hatched area. Altered inputs to V2d, possibly mediated by long-range horizontal connections, are represented by bold arrows. The principal source of signals for either the full or annular wedge condition is identified by the stimulus icons.

ring stimulus, activity in the spared regions of V2d and V3d respond to both signals from the spared foveal signals and from the peripheral signals along the lower vertical meridian. This produces weak eccentric retinotopic organization (Fig. 4B). That we do not see the same colonization of peripheral V2d and V3d in normal controls (Fig. 3) suggests that the activity in G.Y. is mediated by connections that have been strengthened or added through plastic reorganization.

Our results demonstrate that human cortex reorganizes after lesions to primary sensory areas. The principles governing this reorganization can be understood based on simple recruitment of activity by nearby healthy cortex.

\section{REFERENCES}

Barbur JL, Ruddock KH (1980) Spatial characteristics of movement detection mechanisms in human vision. I. Achromatic vision. Biol Cybern 37:77-92.

Barbur JL, Ruddock KH, Waterfield VA (1980) Human visual responses in the absence of the geniculo-calcarine projection. Brain 103:905-928.

Barbur JL, Watson JDG, Frackowiak RSJ, Zeki S (1993) Conscious visual perception without V1. Brain 116:1293-1302.

Berson DM, Stein JJ (1995) Retinotopic organization of the superior colliculus in relation to the retinal distribution of afferent ganglion cells. Vis Neurosci 12:671-686.

Blythe IM, Kennard C, Ruddock KH (1987) Residual vision in patients with retrogeniculate lesions of the visual pathways. Brain 110:887-905.

Boynton GM, Engel SA, Glover GH, Heeger DJ (1996) Linear systems analysis of functional magnetic resonance imaging in human V1. J Neurosci 16:4207-4221.

Brent PJ, Kennard C, Ruddock KH (1994) Residual colour vision in a human hemianope: spectral responses and colour discrimination. Proc R Soc Lond B Biol Sci 256:219-225.

Bullier J, Kennedy H (1983) Projection of the lateral geniculate nucleus onto cortical area V2 in the macaque monkey. Exp Brain Res 53:168-172

Bunt AH, Minckler DS (1977) Foveal sparing. New anatomical evidence for bilateral representation of the central retina. Arch Ophthalmol 95:1445-1447.

Bunt AH, Minckler DS, Johanson GW (1977) Demonstration of bilateral projection of the central retina of the monkey with horseradish peroxidase neuronography. J Comp Neurol 171:619-630.

Buonomano DV, Merzenich MM (1998) Cortical plasticity: from synapses to maps. Annu Rev Neurosci 21:149-186. 
Celesia GG, Bushnell D, Toleikis SC, Brigell MG (1991) Cortical blindness and residual vision: is the "second" visual system in humans capable of more than rudimentary visual perception? Neurology 41:862-869.

Clarke S, Miklossy J (1990) Occipital cortex in man: organization of callosal connections, related myelo- and cytoarchitecture, and putative boundaries of functional visual areas. J Comp Neurol 298:188-214.

Das A, Gilbert CD (1995) Long-range horizontal connections and their role in cortical reorganization revealed by optical recording of cat primary visual cortex. Nature 375:780-784.

DeYoe EA, Carman GJ, Bandettini P, Glickman S, Wieser J, Cox R, Miller D, Neitz J (1996) Mapping striate and extrastriate visual areas in human cerebral cortex. Proc Natl Acad Sci USA 93:2382-2386.

Engel SA, Rumelhart DE, Wandell BA, Lee AT, Glover GH, Chichilnisky EJ, Shadlen MN (1994) fMRI of human visual cortex. Nature 369:525.

Engel SA, Glover GH, Wandell BA (1997) Retinotopic organization in human visual cortex and the spatial precision of functional MRI. Cereb Cortex 7:181-192.

ffytche DH, Guy CN, Zeki S (1996) Motion specific responses from a blind hemifield. Brain 119:1971-1982.

Finlay AL, Jones SR, Morland AB, Ogilvie JA, Ruddock KH (1997) Movement in the normal visual hemifield induces a percept in the "blind" hemifield of a human hemianope. Proc R Soc Lond B Biol Sci 264:267-275

Fries W (1981) The projection from the lateral geniculate nucleus to the prestriate cortex of the macaque monkey. Proc R Soc Lond B Biol Sci 213:73-80.

Gilbert CD (1996) Plasticity in visual perception and physiology. Curr Opin Neurobiol 6:269-274.

Gilbert CD (1998) Adult cortical dynamics. Physiol Rev 78:467-485.

Gilbert CD, Wiesel TN (1992) Receptive field dynamics in adult primary visual cortex. Nature 356:150-152.

Girard P, Bullier J (1989) Visual activity in area V2 during reversible inactivation of area 17 in the macaque monkey. J Neurophysiol 62:1287-1302.

Girard P, Salin PA, Bullier J (1991a) Visual activity in areas V3a and V3 during reversible inactivation of area $\mathrm{V} 1$ in the macaque monkey. J Neurophysiol 66:1493-1503.

Girard P, Salin PA, Bullier J (1991b) Visual activity in macaque area V4 depends on area 17 input. NeuroReport 2:81-84.

Glover GH, Lai S (1998) Self-navigated spiral fMRI: interleaved versus single-shot. Magn Reson Med 39:361-368.

Hadjikhani N, Liu AK, Dale AM, Cavanagh P, Tootell RBH (1998) Retinotopy and color sensitivity in human visual cortical area V8. Nat Neurosci 1:235-241.

Heinen SJ, Skavenski AA (1991) Recovery of visual responses in foveal V1 neurons following bilateral foveal lesions in adult monkey. Exp Brain Res 83:670-674.

Hotson J, Braun D, Herzberg W, Boman D (1994) Transcranial magnetic stimulation of extrastriate cortex degrades human motion direction discrimination. Vision Res 34:2115-2123.

Hubel DH, Wiesel TN (1970) The period of susceptibility to the physiological effects of unilateral eye closure in kittens. J Physiol (Lond) 206:419-436.

Kaas JH, Huerta MF (1988) The subcortical visual system of primates. In: Comparative primate biology, Vol 4, (Steklis HD, Erwin J, eds), pp 327-391. New York: Liss.

Kaas JH, Krubitzer LA, Chino YM, Langston AL, Polley EH, Blair N (1990) Reorganization of retinotopic cortical maps in adult mammals after lesions of the retina. Science 248:229-231.
Kentridge RW, Heywood CA, Weiskrantz L (1997) Residual vision in multiple retinal locations within a scotoma: implications for blindsight. J Cognit Neurosci 9:191-202.

Lucas DR, Baseler HA, Heinen SJ (1996) Eye movements during extended fixation of a stationary target. Invest Ophthalmol Vis Sci 37:S717.

McKeefry DJ, Zeki S (1997) The position and topography of the human colour centre as revealed by functional magnetic resonance imaging. Brain 120:2229-2242.

Meyer CH, Hu BS, Nishimura DG, Macovski A (1992) Fast spiral coronary artery imaging. Magn Reson Med 28:202-213.

Morland AB, Ogilvie JA, Ruddock KH, Wright JR (1996) Orientation discrimination is impaired in the absence of the striate cortical contribution to human vision. Proc R Soc Lond B Biol Sci 263:633-640.

Ogawa S, Lee TM, Kay AR, Tank DW (1990) Brain magnetic resonance imaging with contrast dependent on blood oxygenation. Proc Natl Acad Sci USA 87:9868-9872.

Perenin MT (1991) Discrimination of motion direction in perimetrically blind fields. NeuroReport 2:397-400.

Rodman HR, Gross CG, Albright TD (1989) Afferent basis of visual response properties in area MT of the macaque. I. Effects of striate cortex removal. J Neurosci 9:2033-2050.

Schiller PH, Malpeli JG (1977) The effect of striate cortex cooling on area 18 cells in the monkey. Brain Res 126:366-369.

Sereno MI, Dale AM, Reppas JB, Kwong KK, Belliveau JW, Brady TJ, Rosen BR, Tootell RBH (1995) Borders of multiple visual areas in humans revealed by functional magnetic resonance imaging. Science 268:889-893.

Shatz CJ (1996) Emergence of order in visual system development. Proc Natl Acad Sci USA 93:602-608.

Smith AT, Greenlee MW, Singh KD, Kraemer FM, Hennig J (1998) The processing of first- and second-order motion in human visual cortex assessed by functional magnetic resonance imaging (fMRI). J Neurosci 18:3816-3830.

Standage GP, Benevento LA (1983) The organization of connections between the pulvinar and visual area MT in the macaque monkey. Brain Res 262:288-294.

Teo P, Sapiro G, Wandell BA (1997) Creating connected representations of cortical gray matter for functional MRI visualization. IEEE Med Trans 16:852-863.

Tootell RBH, Mendola JD, Hadjikhani NK, Ledden PJ, Liu AK, Reppas JB, Sereno MI, Dale AM (1997) Functional analysis of V3a and related areas in human visual cortex. J Neurosci 17:7060-7078.

Tootell RBH, Mendola JD, Hadjikhani NK, Liu AK, Dale AM (1998) The representation of the ipsilateral visual field in human cerebral cortex. Proc Natl Acad Sci USA 95:818-824.

Van Essen DC, Newsome WT, Bixby JL (1982) The pattern of interhemispheric connections and its relationship to extrastriate visual areas in the macaque monkey. J Neurosci 2:265-283.

Weiskrantz L, Warrington EK, Sanders MD, Marshall J (1974) Visual capacity in the hemianopic field following a restricted occipital ablation. Brain 97:709-728.

Weiskrantz L, Barbur JL, Sahraie A (1995) Parameters affecting conscious versus unconscious visual discrimination with damage to the visual cortex (V1). Proc Natl Acad Sci USA 92:6122-6126.

Yukie M, Iwai E (1981) Direct projection from the dorsal lateral geniculate nucleus to the prestriate cortex in macaque monkeys. J Comp Neurol 201:81-97.

Zeki SM (1970) Interhemispheric connections of prestriate cortex in monkey. Brain Res 19:63-75. 